\title{
Cosmological aspects of cubic Galileon massive gravity
}

\author{
Shahabeddin M. Aslmarand, ${ }^{1, *}$ Amin Rezaei Akbarieh, ${ }^{2,}{ }^{\dagger}$ Yousef Izadi, ${ }^{3,}{ }^{\ddagger}$ Sobhan Kazempour, ${ }^{4,}$ and Lijing Shao ${ }^{5,}$ \\ ${ }^{1}$ Department of Physics, Florida Atlantic University, Boca Raton, FL 33431, USA \\ ${ }^{2}$ Faculty of Physics, University of Tabriz, Tabriz 51666-16471, Iran \\ ${ }^{3}$ Department of Physics and Applied Physics, University of Massachusetts, Lowell, MA 01854, USA \\ ${ }^{4}$ Faculty of Physics, University of Tabriz, Tabriz 51666-16471, Iran \\ ${ }^{5}$ Kavli Institute for Astronomy and Astrophysics, Peking University, Beijing 100871, China \\ National Astronomical Observatories, Chinese Academy of Sciences, Beijing 100012, China
}

(Dated: November 1, 2021)

\begin{abstract}
Cubic Galileon massive gravity is a development of de Rham-Gabadadze-Tolley (dRGT) massive gravity theory in which the space of the Stueckelberg field is broken. We consider the cubic Galileon term as a scalar field coupled to the graviton field. We present a detailed study of the cosmological aspects of this theory of gravity. We analyze self-accelerating solutions of the background equations of motion to explain the accelerated expansion of the Universe. Exploiting the latest Union2 Type Ia Supernovae (SNIa) dataset, which consists of 557 SNIa, we show that cubic Galileon massive gravity theory is consistent with the observations. We also examine the tensor perturbations within the framework of this model and find an expression for the dispersion relation of gravitational waves, and show that it is consistent with the observational results
\end{abstract}

\section{INTRODUCTION}

Although Einstein's theory of general relativity has been very successful in describing gravity at low energies and explaining observations in Solar system scales, this theory faces fundamental problems in cosmology [15]. General relativity cannot explain the origin of accelerated expansion of the Universe and the cosmological constant problem [6-8]. There are strong observational evidences such as cosmic background radiation (CMB) [9], type Ia supernovae [10] and baryon acoustic oscillations [11] which confirm the existence of these problems. The Universe can be described by the standard models of particle physics and cosmology in microscopic and large cosmological scales, respectively. It is noticeable that many physicists would like to unify these two models into a single comprehensive theory.

One tentative approach for solving the problems in gravity and cosmology is studying a spin-2 massive graviton as a propagator of gravity. Many models have been developed to describe graviton and its interactions. Still, the main concern is finding a theory that would be stable and consistent with observations. It is interesting to note that the first attempt to explain a massive spin-2 particle began by Fierz and Puali in 1939; they proposed a linear action for a massive spin-2 particle (i.e., massive graviton) in a flat space-time [12]. Vainshtein proposed an idea to solve the vDVZ discontinuity, which van Dam, Veltman, and Zakharov found in the limit of the graviton mass $m_{g} \rightarrow 0$ in the linear Fierz-Pauli action, see Refs. [13-15]. Vainshtein argued that to aviod the

\footnotetext{
* smostafanazh2016@fau.edu

$\dagger$ am.rezaei@tabrizu.ac.ir

$\ddagger$ yousef"izadi@uml.edu

$\S$ s.kazempour@tabrizu.ac.ir

『lshao@pku.edu.cn
}

vDVZ discontinuity, Fierz and Pauli's theory should be nonlinear instead of linear. Moreover, in 1979, Boulware and Deser claimed that the nonlinear theory of Fierz and Pauli has a ghost instability which was later called the Boulware-Deser ghost [16].

Eventually, in 2010 de Rham, Gabadadze, and Tolley showed that it is possible to construct a ghost-free nonlinear massive gravity in a certain decoupling limit [17]. They presented a theory with nonlinear interactions which explains massive spin-2 field in a flat spacetime, which is known as a ghost-free dRGT massive gravity theory [18]. However, the dRGT massive gravity theory consists of instabilities in FLRW homogeneous spacetime [19]. So, this issue has motivated to people to propose alternative theories [20-25]. One of the best solutions is using a coupling background to a scalar field which is known as quasi-dilaton massive gravity theory [26]. This is successful in explaining the accelerated expansion of the Universe. However, due to the instability of tensor perturbations in this theory, extensions of it have been introduced to find an improved solution [27, 28].

In this paper, we consider the scalar field to be a cubic Galileon which is coupled to the graviton field. Using the Galileon model, we introduce a scalar field as a candidate for dark energy. Thus, the Galileon model is one of the generalizations of general relativity and can describe the accelerated expansion of the Universe. In this model, the scalar field is invariant under the Galileon transformation $\phi \rightarrow \phi+b_{\mu} x^{\mu}+c$ where $\phi$ is the Galileon field, $b_{\mu}$ and $c$ are constants. The equations of motion obtained from this model are quadratic, and screening occurs via the Vainshtein mechanism. This symmetry was initially proposed in the separation limit of the DvaliGabadadze-Porrati (DGP) brane-world model, which is inherited from Poincaré invariance in higher dimensions $[29,30]$. This symmetry was later used in a more general scalar field theory in which the equations of motion are quadratic. Although Galileons were originally intro- 
duced in the literature of the DGP brane-world model, they also appear in other gravitational models such as massive gravity $[17,18,41]$.

In the recent literature, the word "Galileon" goes far beyond models in which the action is invariant under a symmetric translational transformation, and it is in a class of theories that have derivatives of a coupled field and can establish a consistent cosmological theory. It has been shown in Refs. [31, 32] that Galileons can properly explain dark energy. Also, the results of Refs. [33, 34] show that this model provides an explanation for the origins of the density perturbations in the inflationary era; hence it could even be an alternative to the inflationary models. There has been a tendency towards studying cosmological aspects and perturbation analysis in massive gravity theories and their extensions. For instance, investigation of the constraints on quasi-dilaton massive gravity for finding the bound on graviton mass, see Ref. [35]. Also, dynamical equations, which lead to the expansion history of the Universe throughout all eras in extended quasi-dilaton massive gravity, have been studied in Ref. [36], and in this paper, the effective mass of gravitational waves has been found. Moreover, the new extension of massive gravity theory has been introduced by breaking the translation symmetry in the Stueckelberg space [37]. The perturbation analysis and the propagation of gravitational waves have been studied in the paper as well. Furthermore, some aspects of Galileon in cosmology and modified gravity have been studied [3840]. It is interesting to note that by considering gravitational radiation in binary pulsars in the context of the cubic Galileon massive gravity, a new bound of graviton mass has been obtained in Ref. [41]. Some other valuable studies are in Refs. [42-45].

The outline of this paper is as follows. In Section II we introduce the cubic Galilean massive gravity theory. Also, we obtain the background equations of motion and self-accelerating solutions. In Section III we test the solutions of cubic Galilean massive gravity theory with the latest Union2 type Ia Supernovae (SNIa) dataset, which consists of 557 SNIa. In Section IV we present a perturbation analysis for determining the dispersion relation of gravitational waves in this theory. In Section V, some concluding remarks are given.

\section{THE MODEL}

In this section, we introduce a model in which the scalar field is chosen from the cubic Galileon model. The action of the theory can be written as

$$
\begin{array}{r}
\mathcal{S}=\frac{M_{\mathrm{Pl}}^{2}}{2} \int d^{4} x \sqrt{-g}\{R[g]- \\
2 \Lambda+2 m_{g}^{2}\left(\mathcal{L}_{2}+\alpha_{3} \mathcal{L}_{3}+\alpha_{4} \mathcal{L}_{4}\right) \\
\left.-\frac{\omega}{M_{\mathrm{Pl}}^{2}} \partial_{\mu} \sigma \partial^{\mu} \sigma\left(1+\beta \partial_{\mu} \partial^{\mu} \sigma\right)\right\}
\end{array}
$$

where $M_{\mathrm{Pl}}$ is the reduced Planck mass, $g$ is the determinant of the metric, and $R$ is the Ricci scalar. It is important to note that the kinetic part of the action in eq. (1) is a subclass of a more general action known as the Horndeski action. There is a linear expression in the action of the cubic Galileon [46] that plays the role of potential. Here, we discard this linear term since the theory is invariant under a global dilation of the spacetime coordinates accompanied by a shift of $\sigma . \quad \beta$ is a cubic Galileon parameter; for $\beta=0$ the action in eq. (1) reduces to the standard quasi-dilaton action without any potential term. We assume that $m_{g}$ is a constant parameter, so this model can be considered as a generalization of the quasi-dilaton theory. Part of the action that creates the mass for graviton can be expressed as

$$
\begin{aligned}
\mathcal{L}_{2} & =\frac{1}{2}\left([\mathcal{K}]^{2}-\left[\mathcal{K}^{2}\right]\right), \\
\mathcal{L}_{3} & =\frac{1}{3 !}\left([\mathcal{K}]^{3}-3[\mathcal{K}]\left[\mathcal{K}^{2}\right]+2\left[\mathcal{K}^{3}\right]\right), \\
\mathcal{L}_{4} & =\frac{1}{4 !}\left([\mathcal{K}]^{2}-6[\mathcal{K}]^{2}\left[\mathcal{K}^{2}\right]+3\left[\mathcal{K}^{2}\right]^{2}+8[\mathcal{K}]\left[\mathcal{K}^{3}\right]-6\left[\mathcal{K}^{4}\right][2)\right.
\end{aligned}
$$

where square brackets denote a trace. While these expressions are in a similar form to dRGT theory, in quasidilaton case, the building block tensor $\mathcal{K}$ is defined as

$$
\mathcal{K}_{\nu}^{\mu}=\delta_{\nu}^{\mu}-e^{\frac{\sigma}{M_{\mathrm{Pl}}}}\left(\sqrt{g^{-1} \tilde{g}}\right)_{\nu}^{\mu},
$$

where $\tilde{g}$ is a non-dynamical fiducial metric. This symmetry rules out a non-trivial potential for $\sigma$. Throughout the paper, we adopt the units $c=\hbar=1$ in which the reduced Planck mass becomes $M_{\mathrm{Pl}}=\frac{1}{\sqrt{8 \pi G}}$. Furthermore, we follow the "mostly plus" metric signature convention. Some short-cut notations are used to denote the contractions of rank-2 tensors $\mathcal{K}_{\mu}^{\mu}=[\mathcal{K}]$ and $\mathcal{K}_{\nu}^{\mu} \mathcal{K}_{\mu}^{\nu}=\left[\mathcal{K}^{2}\right]$, etc. Greek indices run from 0 to 3 while Latin indices from 1 to 3 . With the Latin indices we denote contractions in the same way as for the Greek indices $h_{i j} h^{i j}=\left(h_{i j}\right)^{2}, A_{i} A^{i}=\left(A_{i}\right)^{2}$, etc.

For the physical background metric, we use the flat Friedmann-Lemaître-Robertson-Walker (FLRW) ansatz

$$
g_{\mu \nu}=-N(t)^{2} d t^{2}+a(t)^{2} \delta_{i j} d x^{i} d x^{j},
$$

and Minkowski metric is used for non-dynamical fiducial metric denoted by $\tilde{g}_{\mu \nu}$ and expressed as

$$
\tilde{g}_{\mu \nu}=-f^{\prime}(t)^{2} d t^{2}+\delta_{i j} d x^{i} d x^{j} .
$$

Notice that $N$ shows the lapse function of the dynamical metric, and it is similar to a gauge function. The scale factor is represented by $a$, and the dot denotes the derivative with respect to time. In addition, the lapse function $\mathrm{N}$ is related to the coordinate time $t$ and the proper time via $\tau d \tau=N d t[47,48]$. Also, $f(t)$ is the Stueckelberg scalar function whereas $\phi^{0}=f(t)$ and $\partial \phi^{0} / \partial t=\dot{f}(t)$ [49], and unitary gauge corresponds to the choice $f(t)=t$. To obtain the equations of motion, it is better to write eq.

(1) as a minisuperspace action 


$$
\begin{aligned}
\frac{\mathcal{S}}{V}= & \int d t\left\{M_{\mathrm{Pl}}^{2}\left[-3 \frac{a \dot{a}^{2}}{N}-\Lambda a^{3} N\right]+\frac{\omega a^{3}}{2 N} \dot{\sigma}^{2}\left[1-\frac{\beta}{N}\left(\frac{\dot{N}}{N^{2}}-H\right) \dot{\sigma}\right]\right. \\
& +M_{\mathrm{Pl}}^{2} m_{g}^{2}\left[N a^{3}(X-1)\left[3(X-2)-(X-4)(X-1) \alpha_{3}-(X-1)^{2} \alpha_{4}\right]\right. \\
& \left.\left.+f^{\prime} a^{4} X\left((X-1)\left[3-3(X-1) \alpha_{3}+(X-1)^{2} \alpha_{4}\right]\right)\right]\right\}
\end{aligned}
$$

where $V$ is the comoving volume and we used the following definition

$$
X \equiv \frac{e^{\frac{\sigma}{M_{\mathrm{P} 1}}}}{a} .
$$

Note that $X$ is the ratio of scale factors of the metrics $e^{2 \sigma / M_{\mathrm{Pl}}} \tilde{g}_{\mu \nu}$ and $g_{\mu \nu}$. In addition, to further simplify the expressions, we define

$$
H \equiv \frac{\dot{a}}{N a}
$$

where $H$ is the Hubble parameter for the physical metric and

$$
r \equiv \frac{a}{N}
$$

corresponding to the ratio of the speeds of light on these two metrics. Note that we use integration by parts to obtain minisuperspace action (6), so that we convert the second derivative terms into the first order derivatives as follows

$$
\ddot{\sigma} \rightarrow-\frac{\dot{a}}{a}+\frac{\dot{N}}{N} \dot{\sigma}
$$

By varying the minisuperspace action (6) with respect to $f$ and applying unitary gauge condition $f(t)=t$, we have

$$
\frac{d}{d t}\left\{a^{4}(X-1) X\left[3+3 \alpha_{3}+\alpha_{4}-\left(3 \alpha_{3}+2 \alpha_{4}\right) X+\alpha_{4} X^{2}\right]\right\}=0
$$

Integrating the above equation yields

$$
(X-1) X\left[3+3 \alpha_{3}+\alpha_{4}-\left(3 \alpha_{3}+2 \alpha_{4}\right) X+\alpha_{4} X^{2}\right]=\frac{1}{a^{4}} \times C_{0},
$$

where $C_{0}$ is an integral constant. It is worth mentioning that the constant solutions of $X$ lead to the effective energy density, and shows similar behavior to a cosmological constant. In an expanding Universe, the scale factor grows as time passes, so the right-hand side of that eq. (12) decreases. Therefore, after a long enough time, $X$ leads to a constant value $X_{\mathrm{SA}}$ which makes the left-hand side of the equation zero. One of the solutions for the eq. (12) is $X=0$ which leads to $\sigma \rightarrow 0$. Meanwhile, this solution multiplies to the perturbations of the auxiliary scalars, which means that we encounter strong coupling in the vector and scalar sectors. Thus, to avoid strong coupling, we discard this solution [26]. Therefore, we have

$$
\left.(X-1) X\left[3+3 \alpha_{3}+\alpha_{4}-\left(3 \alpha_{3}+2 \alpha_{4}\right) X+\alpha_{4} X^{2}\right]\right|_{X=X_{\mathrm{SA}}}=0
$$

Another solution is $X=1$, which leads to a vanishing cosmological constant and because of inconsistency, it is unacceptable and so it should be ignored too [26]. As a result, the two remaining solutions of the eq. (13) are

$$
X_{\mathrm{SA}}^{ \pm}=\frac{3 \alpha_{3}+2 \alpha_{4} \pm \sqrt{9 \alpha_{3}^{2}-12 \alpha_{4}}}{2 \alpha_{4}} .
$$

Using this result, we can find the modified Friedmann 


$$
\begin{aligned}
3 H^{2}-\frac{\omega}{2 M_{\mathrm{P} 1}^{2}} \frac{\dot{\sigma}^{2}}{N^{2}}\left(1-3 \beta \frac{\ddot{\sigma}}{N^{2}}\right)=\Lambda+ & m_{g}^{2}\left\{\left(\alpha_{3}+\alpha_{4}\right) X^{3}-3\left(1+2 \alpha_{3}+\alpha_{4}\right) X^{2}\right. \\
& \left.+3\left(3+3 \alpha_{3}+\alpha_{4}\right) X-\left(6+4 \alpha_{3}+\alpha_{4}\right)\right\},
\end{aligned}
$$

where $\frac{\dot{\sigma}}{N}=M_{\mathrm{Pl}}\left(H+\frac{\dot{X}}{N X}\right)$ and $\frac{\ddot{\sigma}}{N^{2}}=\frac{M_{\mathrm{Pl}}}{N^{2}} \frac{d}{d t}\left(N H+\frac{\dot{X}}{X}\right)$. If $X=X_{\mathrm{SA}}^{ \pm}$, using time re-parametrization invariance to set $N(t)=1$, we obtain the Friedmann equation

$$
\left[3-\frac{\omega}{2}\left(1-3 \beta M_{\mathrm{Pl}} \dot{H}\right)\right] H^{2}=\Lambda+\xi^{ \pm}
$$

Here $\xi^{ \pm}$is a fixed quantity, which is defined as follows

$$
\begin{aligned}
\xi^{ \pm}= & m_{g}^{2}\left\{\left(\alpha_{3}+\alpha_{4}\right)\left(X_{\mathrm{SA}}^{ \pm}\right)^{3}-3\left(1+2 \alpha_{3}+\alpha_{4}\right)\left(X_{\mathrm{SA}}^{ \pm}\right)^{2}\right. \\
& \left.+3\left(3+3 \alpha_{3}+\alpha_{4}\right)\left(X_{\mathrm{SA}}^{ \pm}\right)^{3}-\left(6+4 \alpha_{3}+\alpha_{4}\right)\right\} \cdot(17)
\end{aligned}
$$

One can see that $\xi^{ \pm}$has appeared as an additional cosmological constant in Friedmann's equation. For describ- ing the dynamics of the Hubble parameter, it is better to convert time in eq. (16) to the redshift. Applying change of variable $\frac{d}{d t}=-H(z+1) \frac{d}{d z}$, the modified Friedmann equation becomes

$$
\left[3-\frac{\omega}{2}\left(1+3 \beta M_{\mathrm{Pl}} H(1+z) H^{\prime}\right)\right] H^{2}=\Lambda+\xi^{ \pm},
$$

where prime denotes the derivative with respect to the redshift $z$. For the case $\beta=0$, the Friedmann equation (18) provides a condition on the parameter $\omega$. For the self-accelerating solutions, to keep the left hand side of the Friedmann equation (18) positive, one needs to have $\omega<6$. This ensures having standard cosmology during matter domination if we add the ordinary matter to the right-hand side of the equation. For the case $\beta \neq 0$, if $\omega$ is equal to 6 , the solution to the asymptotic state of eq. (18) in small redshifts can be obtained as

$$
H(z) \sim H_{0}+\frac{\zeta^{ \pm}}{H_{0}^{3}} z+\frac{\left[-3\left(\zeta^{ \pm}\right)^{2}-\zeta^{ \pm} H_{0}^{4}\right]}{2 H_{0}^{7}} z^{2}+\frac{\zeta^{ \pm}\left[21\left(\zeta^{ \pm}\right)^{2}+9 \zeta^{ \pm} H_{0}^{4}+2 H_{0}^{8}\right]}{6 H_{0}^{11}} z^{3}+\ldots
$$

where $\zeta^{ \pm}$is defined as

$$
\zeta^{ \pm} \equiv-\frac{2}{9} \frac{\Lambda+\xi^{ \pm}}{\beta M_{\mathrm{Pl}}},
$$

and $H_{0}$ represents the Hubble parameter at the present time. In the case $\omega=6$, one can conclude that for any value of $\beta$, the Hubble parameter is well behaved and so this model can explain the accelerated expansion of the Universe.

Taking the variation of action (6) with respect to the scalar field, the equation of motion corresponding to $\sigma$ is obtained as

$$
\begin{aligned}
0 & =3 H\left(H+\frac{\dot{X}}{N X}\right)+\frac{1}{N} \frac{d}{d t}\left(H+\frac{\dot{X}}{N X}\right)+\frac{3}{2} \beta\left(H+\frac{\dot{X}}{N X}\right) \Xi-\frac{m_{g}^{2}}{\omega} X \\
& \times\left\{-(3+r)\left(3+3 \alpha_{3}+\alpha_{4}\right)+6(1+r)\left(1+2 \alpha_{3}+\alpha_{4}\right) X-3(1+3 r)\left(\alpha_{3}+\alpha_{4}\right) X^{2}+4 r \alpha_{4} X\right\}
\end{aligned}
$$

where

$$
\begin{aligned}
\Xi \equiv & M_{\mathrm{Pl}}\left\{3 H^{2}+\frac{\dot{H}}{N}+\frac{\dot{N}^{2}}{N^{4}}-5 H \frac{\dot{N}}{N^{2}}-\frac{\ddot{N}}{N^{3}}\right\}\left(H+\frac{\dot{X}}{N X}\right) \\
& +2 M_{\mathrm{Pl}}\left(H-\frac{\dot{N}}{N^{2}}\right) \frac{1}{N^{2}} \frac{d}{d t}\left(N H+\frac{\dot{X}}{X}\right) .
\end{aligned}
$$


Exploiting eq. (21), one finds

$$
\begin{aligned}
r_{\mathrm{SA}} & =\frac{1}{m_{g}^{2}(\omega-6)\left(X_{\mathrm{SA}}^{ \pm}\right)^{2}\left(X_{\mathrm{SA}}^{ \pm} \alpha_{3}-\alpha_{3}-2\right)}\left\{2\left(m_{g}^{2}\left(3+\alpha_{3}\right)-\Lambda\right) \omega-2 \beta(\omega-6) \omega H^{4} M_{\mathrm{Pl}}^{2}\right. \\
& \left.+m_{g}^{2} X_{\mathrm{SA}}^{ \pm}\left[X_{\mathrm{SA}}^{ \pm}\left(12+6 \alpha_{3}+4 \omega+5 \alpha_{3} \omega-X_{\mathrm{SA}}^{ \pm} \alpha_{3}(\omega+6)\right)-6 \omega\left(\alpha_{3}+2\right)\right]\right\} .
\end{aligned}
$$

\section{COSMOLOGICAL TESTS}

In 1998, observations on distant type Ia supernovae confirmed the accelerated expansion of the Universe [5053]. In this section, using the Union2 SNIa dataset consisting of 557 SNIa [54], we examine the cubic Galileon massive gravity model. The results of the Union2 SNIa dataset can be expressed in terms of $\mu_{\mathrm{obs}}$, and should be compared with the predictions of the model

$$
\mu_{\mathrm{th}}\left(z_{i}\right)=5 \log _{10} D_{L}\left(z_{i}\right)+\mu_{0},
$$

where $\mu_{0}=42.38-5 \log _{10} h$ ( $h$ is the Hubble constant $H_{0}$ in units of $100 \mathrm{~km} / \mathrm{s} / \mathrm{Mpc}$ ), and

$$
D_{L}(z)=(1+z) \int_{0}^{z} \frac{d x}{E(x ; p)},
$$

where $E=H / H_{0}$ and $p$ denotes the model parameters. It should be noted that $X^{2}$ from the 557 Union2 SNIa is given by

$$
X_{\mu}^{2}(p)=\sum_{i} \frac{\left[\mu_{\mathrm{obs}}\left(z_{i}\right)-\mu_{\mathrm{th}}\left(z_{i}\right)\right]^{2}}{\sigma^{2}\left(z_{i}\right)}
$$

where $\sigma$ is related to $1 \sigma$ error and the parameter $\mu_{0}$ is a nuisance parameter and is independent of the data points. According to Refs. [55, 56], we expand $X_{\mu}^{2}$ in eq. (26) to minimize it with respect to $\mu_{0}$,

$$
X_{\mu}^{2}(p)=\tilde{A}-2 \mu_{0} \tilde{B}+\mu_{0}^{2} \tilde{C},
$$

where

$$
\begin{aligned}
& \tilde{A}(p)=\sum_{i} \frac{\left[\mu_{\mathrm{obs}}\left(z_{i}\right)-\mu_{\mathrm{th}}\left(z_{i} ; \mu_{0}=0, p\right)\right]^{2}}{\sigma_{\mu_{\mathrm{obs}}}^{2}\left(z_{i}\right)}, \\
& \tilde{B}(p)=\sum_{i} \frac{\mu_{\mathrm{obs}}\left(z_{i}\right)-\mu_{\mathrm{th}}\left(z_{i} ; \mu_{0}=0, p\right)}{\sigma_{\mu_{\mathrm{obs}}}^{2}\left(z_{i}\right)}, \\
& \tilde{C}=\sum_{i} \frac{1}{\sigma_{\mu_{\mathrm{obs}}}^{2}\left(z_{i}\right)} .
\end{aligned}
$$

For $\mu_{0}=\frac{\tilde{B}}{\tilde{C}}$, eq. (27) has a minimum at

$$
\tilde{X}_{\mu}^{2}(p)=\tilde{A}(p)-\frac{\tilde{B}^{2}(p)}{\tilde{C}} .
$$

Since $X_{\mu, \min }^{2}=\tilde{X}_{\mu, \min }^{2}$, we can consider minimizing $\tilde{X}_{\mu}^{2}$ which is independent of $\mu_{0}$. It is important to note that the best-fit model parameters are determined by minimizing $X^{2}=\tilde{X}_{\mu}^{2}$. Clearly, the corresponding $h$ can be determined by $\mu_{0}=\frac{\tilde{B}}{\tilde{C}}$ for the best-fit parameters. From eq. (19), the dimensionless Hubble parameter can be written as

$$
E=\frac{H(z)}{H_{0}}=1+Y z-\left(\frac{3 Y^{2}+Y}{2}\right) z^{2}+\left(\frac{21 Y^{3}+9 Y^{2}+2 Y}{6}\right) z^{3}+\ldots
$$

where

$$
Y=\frac{\zeta^{ \pm}}{H_{0}^{4}}
$$

We plot the corresponding $X^{2}$ and likelihood as functions of parameter $Y$ in Fig. 1. The best fit has $X_{\min }^{2}=$ 543.579 , and the best-fit parameter is

$$
\begin{aligned}
& Y=0.394_{-0.131}^{+0.147}, \quad \text { with } 1 \sigma \text { uncertainty, } \\
& Y=0.394_{-0.250}^{+0.312}, \quad \text { with } 2 \sigma \text { uncertainty. }
\end{aligned}
$$

Furthermore, the best fit for the Hubble parameter in this theory is $h=0.701$. In Fig. 2, we illustrate the Hubble diagram for the best fit in comparison with the 557 Union2 SNIa data points. One can see that the cubic Galileon massive gravity is well consistent with the 557 Union2 SNIa dataset.

\section{TENSOR PERTURBATIONS}

In this section, we analyze tensor perturbation to calculate the dispersion relation of gravitational waves. The first step is to find the action in the second order of the perturbations. For this, we consider small fluctuations 


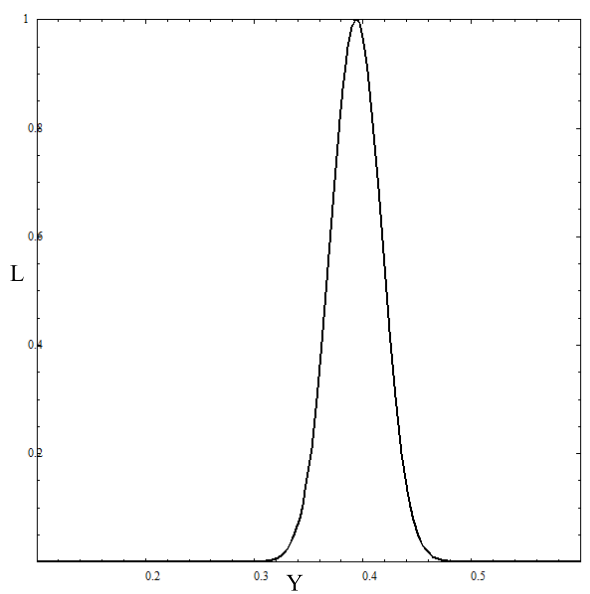

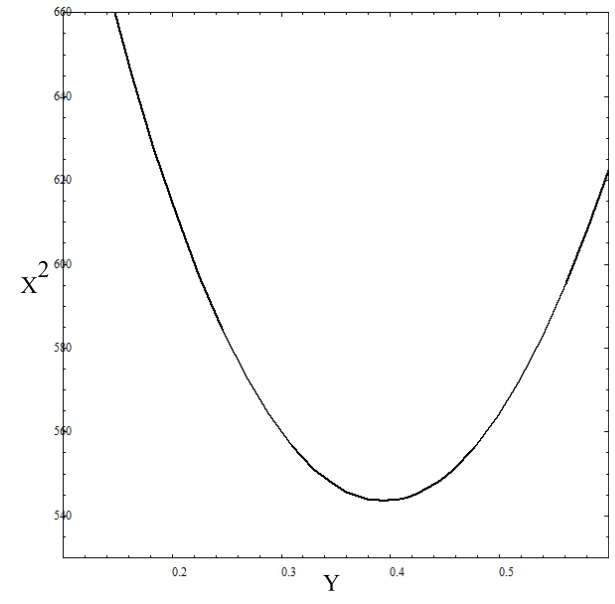

FIG. 1. The $X^{2}$ and likelihood as functions of parameter $Y$.

$\delta g_{\mu \nu}$, and expand the physical metric around a back- ground solution $g_{\mu \nu}^{(0)}$,

$$
g_{\mu \nu}=g_{\mu \nu}^{(0)}+\delta g_{\mu \nu}
$$

We require the terms in quadratic order in $\delta g_{\mu \nu}$ to be kept. It should be noted that we perform all calculations in the unitary gauge, so we do not worry about the form of gauge-invariant combinations. Here, we raise and lower the spatial indices on perturbations by $\delta^{i j}$ and $\delta_{i j}$. Moreover, we can write the expanded action in the Fourier domain with plane waves, i.e., with replacements $\vec{\nabla}^{2} \rightarrow-k^{2}$ and $d^{3} x \rightarrow d^{3} k$. Note that we take $N=1$,

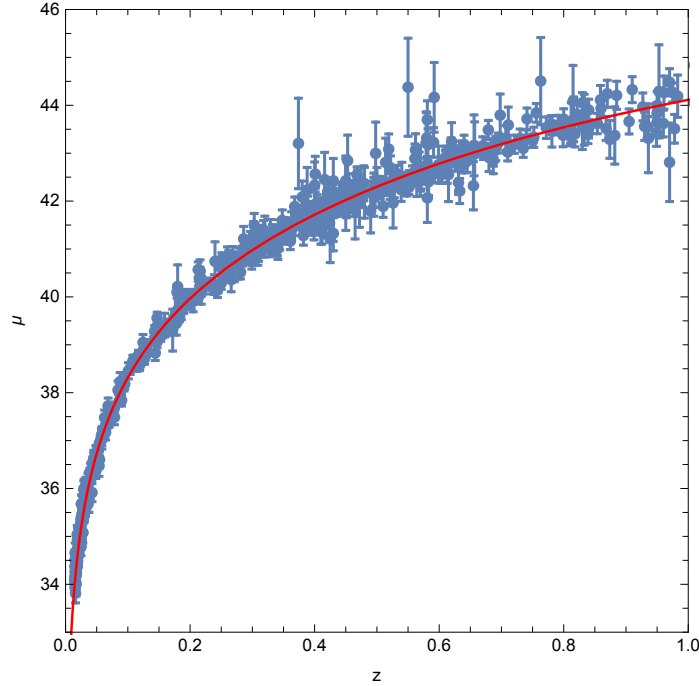

FIG. 2. The Hubble diagram for the best fit (red solid line), comparing with the 557 Union2 SNIa data points (blue dots).

which means that the derivatives should be considered with respect to time in the following calculations.

Let us begin by considering tensor perturbations as

$$
\delta g_{i j}=a^{2} h_{i j}^{T T},
$$

where

$$
\partial^{i} h_{i j}=0, \quad g^{i j} h_{i j}=0 .
$$

The tensor perturbed action in the second order can be calculated for each part of the action separately. Gravity part of the perturbed action in quadratic order is

$$
S_{\text {gravity }}^{(2)}=\frac{M_{\mathrm{Pl}}^{2}}{8} \int d^{3} k d t a^{3}\left[\dot{h}_{i j} \dot{h}^{i j}-\left(\frac{k^{2}}{a^{2}}+4 \dot{H}+6 H^{2}-2 \Lambda\right) h^{i j} h_{i j}\right] .
$$

The second order piece of massive gravity sector of the perturbed action can be written as

$$
\begin{aligned}
S_{\text {massive }}^{(2)}= & \frac{M_{\mathrm{Pl}}^{2}}{8} \int d^{3} k d t a^{3} m_{g}^{2}\left[\left(\alpha_{3}+\alpha_{4}\right) r X^{3}-\left(1+2 \alpha_{3}+\alpha_{4}\right)(1+3 r) X^{2}\right. \\
& \left.+\left(3+3 \alpha_{3}+\alpha_{4}\right)(3+2 r) X-2\left(6+4 \alpha_{3}+\alpha_{4}\right)\right] h^{i j} h_{i j} .
\end{aligned}
$$


In addition, we write cubic Galileon part of the perturbed action in quadratic order

$$
S_{\text {cubic-Galileon }}^{(2)}=-\frac{M_{\mathrm{Pl}}^{2}}{8} \int d^{3} k d t a^{3}\left[\frac{\omega}{M_{\mathrm{Pl}}^{2}} \dot{\sigma}^{2}\left(1-\beta \dot{\sigma}^{2}\right) h^{i j} h_{i j}\right] .
$$

Summing up the second order pecies of the perturbed actions $S_{\text {gravity }}^{(2)}, S_{\text {massive }}^{(2)}$ and $S_{\text {cubic-Galileon }}^{(2)}$, we obtain the total action in second order for tensor perturbations

$$
S_{\text {total }}^{(2)}=\frac{M_{\mathrm{Pl}}^{2}}{8} \int d^{3} k d t a^{3}\left\{\dot{h}^{i j} \dot{h}_{i j}-\left(\frac{k^{2}}{a^{2}}+M_{\mathrm{GW}}^{2}\right) h^{i j} h_{i j}\right\} .
$$

At this point, using eqs. (14) and (23) we calculate $\alpha_{3}$ and $\alpha_{4}$. Therefore, the dispersion relation of gravitational waves is obtained as

$$
M_{\mathrm{GW}}^{2}=4 \dot{H}+6 H^{2}+\frac{\omega}{M_{\mathrm{Pl}}^{2}} \dot{\sigma}^{2}\left(1-\beta \dot{\sigma}^{2}\right)+\gamma
$$

where

$$
\begin{aligned}
\gamma= & \frac{1}{\left(X_{\mathrm{SA}}^{ \pm}-1\right)\left[2 \omega-4 \omega X_{\mathrm{SA}}^{ \pm}+(6+\omega)\left(X_{\mathrm{SA}}^{ \pm}\right)^{2}\right.} \times \\
& \left\{2 \beta(\omega-6) \omega H^{4} M_{\mathrm{Pl}}^{2}\left[\left(X_{\mathrm{SA}}^{ \pm}-3\right) X_{\mathrm{SA}}^{ \pm}\left(r_{\mathrm{SA}} X_{\mathrm{SA}}^{ \pm}-2\right)-2\right]\right. \\
& +X_{\mathrm{SA}}^{ \pm}\left[6\left(m_{g}^{2}-\Lambda\right)\left(\omega\left(r_{\mathrm{SA}}-1\right)-2\right)+X_{\mathrm{SA}}^{ \pm}\left(2 \Lambda\left(\omega\left(r_{\mathrm{SA}}-1\right)-6\right)+12 m_{g}^{2}\left(3+\omega-r_{\mathrm{SA}} \omega\right)\right.\right. \\
& \left.\left.\left.+m_{g}^{2} X_{\mathrm{SA}}^{ \pm}\left[6 \omega\left(r_{\mathrm{SA}}-1\right)+X_{\mathrm{SA}}^{ \pm}\left(6+6 r_{\mathrm{SA}}+\omega-r_{\mathrm{SA}} \omega\right)-36\right]\right)\right]\right\} .
\end{aligned}
$$

It is interesting to note that if the square of the mass of gravitational waves is positive, the stability of longwavelength gravitational waves is guaranteed. However, if it is negative, it should be tachyonic. Hence, as the mass of the tachyon is of the order of the Hubble scale, the instability should take the age of the Universe to develop.

\section{CONCLUSION}

In this work we have presented the cubic Galileon massive gravity theory which is a development of de RhamGabadadze-Tolley (dRGT) massive gravity theory. We have introduced the action and have found the full set of equations of motion for a FLRW background. To explain the late-time acceleration of the Universe, we have analyzed the self-accelerating background solutions.

In addition, we have tested the solution of cubic Galileon massive gravity theory with the latest Union2 SNIa dataset, which consists of 557 SNIa, and have demonstrated the compatibility of the model with the observational data. Therefore, this comparison with observational data for the late-time acceleration of the Universe can be very useful for checking the parameters of the the cubic Galileon massive gravity theory. We have illustrated the Hubble diagram for the best fit in comparison with the 557 Union2 SNIa data points and the best fit for the Hubble parameter in this theory is $h=0.701$.

In the last section of this paper, for examining the mass of graviton in the framework of cubic Galileon massive gravity theory, we have presented a detailed analysis of tensor perturbation and have obtained the dispersion relation of gravitational waves. We have studied the propagation of gravitational perturbation in the FLRW cosmology in the cubic Galileon massive gravity theory. This kind of analysis is crucial for probing the alternative gravity theories in the era of gravitational waves.

\section{ACKNOWLEDGMENTS}

We are really grateful to Nishant Agarwal for his very useful notes and codes on tensor perturbations. ARA, YI and SK would like to thank A. Emir Gumrukcuolu and Hao Wei for useful discussions and comments. LS was supported by the National Natural Science Foundation of China (Grants No. 11975027, No. 11991053, No. 11721303), the National SKA Program of China (No. 2020SKA0120300), and the Max Planck Partner Group Program funded by the Max Planck Society.
[1] C. M. Will, Living Rev. Rel. 4 (2001), 4 doi:10.12942/lrr2001-4 [arXiv:gr-qc/0103036 [gr-qc]].
[2] S. Reynaud and M. T. Jaekel, Proc. Int. Sch. Phys. Fermi 168 (2009), 203-217 doi:10.3254/978-1-58603-990-5-203 
[arXiv:0801.3407 [gr-qc]].

[3] C. W. F. Everitt, D. B. DeBra, B. W. Parkinson, J. P. Turneaure, J. W. Conklin, M. I. Heifetz, G. M. Keiser, A. S. Silbergleit, T. Holmes and J. Kolodziejczak, et al. Phys. Rev. Lett. 106 (2011), 221101 doi:10.1103/PhysRevLett.106.221101 [arXiv:1105.3456 [gr-qc]].

[4] E. Berti, E. Barausse, V. Cardoso, L. Gualtieri, P. Pani, U. Sperhake, L. C. Stein, N. Wex, K. Yagi and T. Baker, et al. Class. Quant. Grav. 32 (2015), 243001 doi:10.1088/0264-9381/32/24/243001 [arXiv:1501.07274 [gr-qc]].

[5] N. Arkani-Hamed, S. Dimopoulos and G. R. Dvali, Phys. Lett. B 429 (1998), 263-272 doi:10.1016/S03702693(98)00466-3 [arXiv:hep-ph/9803315 [hep-ph]].

[6] S. Weinberg, Rev. Mod. Phys. 61 (1989), 1-23 doi:10.1103/RevModPhys.61.1

[7] P. J. E. Peebles and B. Ratra, Rev. Mod. Phys. 75 (2003), 559-606 doi:10.1103/RevModPhys.75.559 [arXiv:astro$\mathrm{ph} / 0207347$ [astro-ph]].

[8] A. G. Riess et al. [Supernova Search Team], Astron. J. 116 (1998), 1009-1038 doi:10.1086/300499 [arXiv:astro$\mathrm{ph} / 9805201$ [astro-ph]].

[9] P. A. R. Ade et al. [Planck], Astron. Astrophys. 594 (2016), A13 doi:10.1051/0004-6361/201525830 [arXiv:1502.01589 [astro-ph.CO]].

[10] M. M. Phillips, Astrophys. J. Lett. 413 (1993), L105L108 doi:10.1086/186970

[11] F. Beutler, C. Blake, M. Colless, D. H. Jones, L. Staveley-Smith, L. Campbell, Q. Parker, W. Saunders and F. Watson, Mon. Not. Roy. Astron. Soc. 416 (2011), 3017-3032 doi:10.1111/j.1365-2966.2011.19250.x [arXiv:1106.3366 [astro-ph.CO]].

[12] M. Fierz and W. Pauli, Proc. Roy. Soc. Lond. A 173 (1939), 211-232 doi:10.1098/rspa.1939.0140

[13] A. I. Vainshtein, Phys. Lett. B 39 (1972), 393-394 doi:10.1016/0370-2693(72)90147-5

[14] H. van Dam and M. J. G. Veltman, Nucl. Phys. B 22 (1970), 397-411 doi:10.1016/0550-3213(70)90416-5

[15] V. I. Zakharov, JETP Lett. 12 (1970), 312

[16] D. G. Boulware and S. Deser, Phys. Rev. D 6 (1972), 3368-3382 doi:10.1103/PhysRevD.6.3368

[17] C. de Rham and G. Gabadadze, Phys. Rev. D 82 (2010), 044020 doi:10.1103/PhysRevD.82.044020 [arXiv:1007.0443 [hep-th]].

[18] C. de Rham, G. Gabadadze and A. J. Tolley, Phys. Rev. Lett. $106 \quad$ (2011), 231101 doi:10.1103/PhysRevLett.106.231101 [arXiv:1011.1232 [hep-th]].

[19] A. De Felice, A. E. Gumrukcuoglu and S. Mukohyama, Phys. Rev. Lett. 109 (2012), 171101 doi:10.1103/PhysRevLett.109.171101 [arXiv:1206.2080 [hep-th]].

[20] G. D'Amico, C. de Rham, S. Dubovsky, G. Gabadadze, D. Pirtskhalava and A. J. Tolley, Phys. Rev. D 84 (2011), 124046 doi:10.1103/PhysRevD.84.124046 [arXiv:1108.5231 [hep-th]].

[21] A. E. Gumrukcuoglu, C. Lin and S. Mukohyama, Phys. Lett. B $\mathbf{7 1 7}$ (2012), 295-298 doi:10.1016/j.physletb.2012.09.049 [arXiv:1206.2723 [hep-th]].

[22] A. De Felice, A. E. Gümrükçüoğlu, C. Lin and S. Mukohyama, JCAP 05 (2013), 035 doi:10.1088/14757516/2013/05/035 [arXiv:1303.4154 [hep-th]].
[23] Q. G. Huang, Y. S. Piao and S. Y. Zhou, Phys. Rev. D 86 (2012), 124014 doi:10.1103/PhysRevD.86.124014 [arXiv:1206.5678 [hep-th]].

[24] S. F. Hassan and R. A. Rosen, JHEP 02 (2012), 126 doi:10.1007/JHEP02(2012)126 [arXiv:1109.3515 [hepth]].

[25] K. Hinterbichler and R. A. Rosen, JHEP 07 (2012), 047 doi:10.1007/JHEP07(2012)047 [arXiv:1203.5783 [hepth]].

[26] G. D'Amico, G. Gabadadze, L. Hui and D. Pirt$\begin{array}{lllll}\text { skhalava, Phys. Rev. D } 87 & \text { (2013), } 064037\end{array}$ doi:10.1103/PhysRevD.87.064037 [arXiv:1206.4253 [hep-th]].

[27] A. De Felice and S. Mukohyama, Phys. Lett. B 728 (2014), 622-625 doi:10.1016/j.physletb.2013.12.041 [arXiv:1306.5502 [hep-th]].

[28] S. Mukohyama, JCAP 12 (2014), 011 doi:10.1088/14757516/2014/12/011 [arXiv:1410.1996 [hep-th]].

[29] M. A. Luty, M. Porrati and R. Rattazzi, JHEP 09 (2003), 029 doi:10.1088/1126-6708/2003/09/029 [arXiv:hep-th/0303116 [hep-th]].

[30] G. R. Dvali, G. Gabadadze and M. Porrati, Phys. Lett. B 484 (2000), 112-118 doi:10.1016/S0370-2693(00)00631-6 [arXiv:hep-th/0002190 [hep-th]].

[31] T. Kobayashi, Phys. Rev. D 81 (2010), 103533 doi:10.1103/PhysRevD.81.103533 [arXiv:1003.3281 [astro-ph.CO]].

[32] N. Chow and J. Khoury, Phys. Rev. D 80 (2009), 024037 doi:10.1103/PhysRevD.80.024037 [arXiv:0905.1325 [hepth]].

[33] C. Germani, Phys. Dark Univ. 15 (2017), 1-6 doi:10.1016/j.dark.2016.11.003 [arXiv:1609.06598 [astroph.CO]].

[34] C. Burrage, C. de Rham, D. Seery and A. J. Tolley, JCAP 01 (2011), 014 doi:10.1088/1475-7516/2011/01/014 [arXiv:1009.2497 [hep-th]].

[35] K. Martinovic and M. Sakellariadou, Phys. Rev. D 100 (2019) no.12, 124016 doi:10.1103/PhysRevD.100.124016 [arXiv:1908.08247 [gr-qc]].

[36] T. Kahniashvili, A. Kar, G. Lavrelashvili, N. Agarwal, L. Heisenberg and A. Kosowsky, Phys. Rev. D 91 (2015) no.4, 041301 [erratum: Phys. Rev. D 100 (2019) no.8, 089902] doi:10.1103/PhysRevD.91.041301 [arXiv:1412.4300 [astro-ph.CO]].

[37] M. Kenna-Allison, A. E. Gumrukcuoglu and K. Koyama, Phys. Rev. D 102 (2020) no.10, 103524 doi:10.1103/PhysRevD.102.103524 [arXiv:2009.05405 [gr-qc]].

[38] A. Nicolis, R. Rattazzi and E. Trincherini, Phys. Rev. D 79 (2009), 064036 doi:10.1103/PhysRevD.79.064036 [arXiv:0811.2197 [hep-th]].

[39] C. de Rham and L. Heisenberg, Phys. Rev. D 84 (2011), 043503 doi:10.1103/PhysRevD.84.043503 [arXiv:1106.3312 [hep-th]].

[40] M. Andrews, G. Goon, K. Hinterbichler, J. Stokes and M. Trodden, Phys. Rev. Lett. 111 (2013) no.6, 061107 doi:10.1103/PhysRevLett.111.061107 [arXiv:1303.1177 [hep-th]].

[41] L. Shao, N. Wex and S. Y. Zhou, Phys. Rev. D 102 (2020) no.2, 024069 doi:10.1103/PhysRevD.102.024069 [arXiv:2007.04531 [gr-qc]].

[42] A. E. Gumrukcuoglu, R. Kimura and K. Koyama, $\begin{array}{lllll}\text { Phys. Rev. D } 101 & \text { (2020) no.12, } 124021\end{array}$ doi:10.1103/PhysRevD.101.124021 [arXiv:2003.11831 
[gr-qc]].

[43] A. R. Akbarieh, S. Kazempour and L. Shao, Phys. Rev. D 103 (2021), 123518 doi:10.1103/PhysRevD.103.123518 [arXiv:2105.03744 [gr-qc]].

[44] A. E. Gümrükçüoğlu, K. Hinterbichler, C. Lin, S. Mukohyama and M. Trodden, Phys. Rev. D 88 (2013) no.2, 024023 doi:10.1103/PhysRevD.88.024023 [arXiv:1304.0449 [hep-th]].

[45] P. Guarato and R. Durrer, Phys. Rev. D 89 (2014) no.8, 084016 doi:10.1103/PhysRevD.89.084016 [arXiv:1309.2245 [gr-qc]].

[46] J. Zhang, B. R. Dinda, M. W. Hossain, A. A. Sen and W. Luo, Phys. Rev. D 102 (2020) no.4, 043510 doi:10.1103/PhysRevD.102.043510 [arXiv:2004.12659 [astro-ph.CO]].

[47] M. A. Scheel, S. L. Shapiro and S. A. Teukolsky, Phys. Rev. D 51 (1995) 4236 doi:10.1103/PhysRevD.51.4236 [gr-qc/9411026].

[48] T. Christodoulakis, N. Dimakis and P. A. Terzis, J. Phys. A 47 (2014) 095202 doi:10.1088/1751-8113/47/9/095202 [arXiv:1304.4359 [gr-qc]].

[49] N. Arkani-Hamed, H. Georgi and M. D. Schwartz, Annals Phys. 305 (2003) 96 doi:10.1016/S0003-4916(03)00068-X [hep-th/0210184].

[50] E. J. Copeland, M. Sami and S. Tsujikawa, Int. J. Mod. Phys. D 15 (2006) 1753 doi:10.1142/S021827180600942X [hep-th/0603057].

[51] J. Frieman, M. Turner and D. Huterer, Ann. Rev. Astron. Astrophys. $46 \quad$ (2008) 385 doi:10.1146/annurev.astro.46.060407.145243 [arXiv:0803.0982 [astro-ph]].

[52] S. Perlmutter and B. P. Schmidt, Lect. Notes Phys. 598 (2003) 195 doi:10.1007/3-540-45863-8-11 [astro$\mathrm{ph} / 0303428]$.

[53] Y. Yang and Y. Gong, JCAP 2006 (2020) 059 doi:10.1088/1475-7516/2020/06/059 [arXiv:1912.07375 [astro-ph.CO]].

[54] R. Amanullah et al., Astrophys. J. 716 (2010) 712 doi:10.1088/0004-637X/716/1/712 [arXiv:1004.1711 [astro-ph.CO]].

[55] S. Nesseris and L. Perivolaropoulos, Phys. Rev. D 72 (2005), 123519 doi:10.1103/PhysRevD.72.123519 [arXiv:astro-ph/0511040 [astro-ph]].

[56] E. Di Pietro and J. F. Claeskens, Mon. Not. Roy. Astron. Soc. 341 (2003), 1299 doi:10.1046/j.13658711.2003.06508.x [arXiv:astro-ph/0207332 [astro-ph]]. 